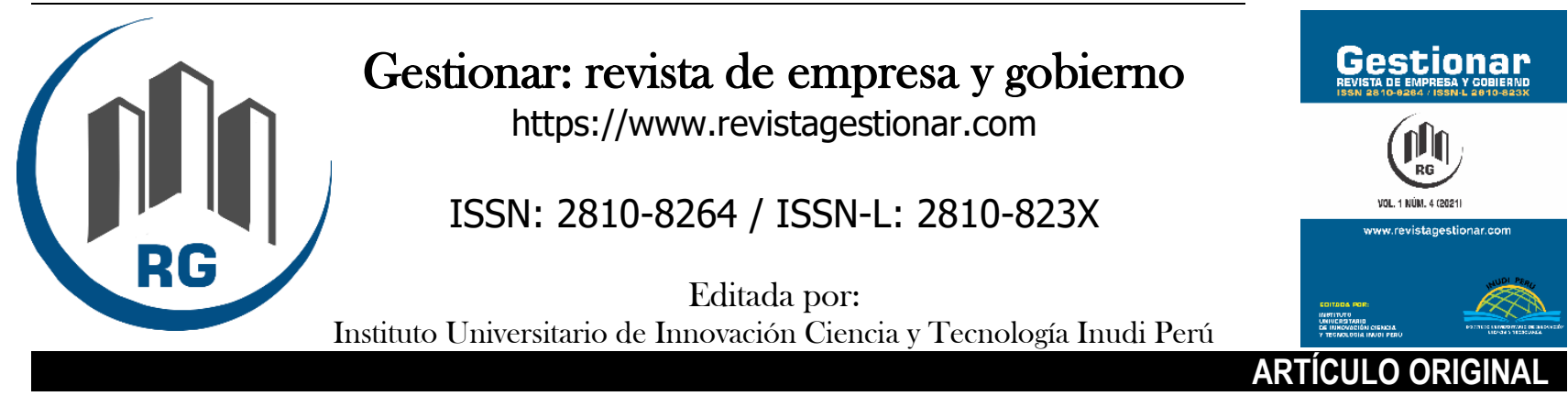

\title{
Deserción estudiantil en el contexto de la COVID-19: alternativas para la retención universitaria
}

\author{
Student dropout in the context of COVID-19: alternatives for university retention
}

A evasão estudantil no contexto da COVID-19: alternativas para a retenção universitária

\author{
María Seminara ${ }^{1}$ \\ Universidad Nacional de San Juan, San Juan, Argentina \\ (iD https://orcid.org/0000-0003-1823-0034 \\ mseminara@unsj.edu.ar
}

\author{
Arnoldo Fernández \\ Universidad Nacional de San Juan, San Juan, Argentina \\ (iD https://orcid.org/0000-0003-3027-0450 \\ afernandez@ unsj.edu.ar
}

\author{
Alejandra Pérez \\ Universidad Nacional de San Juan, San Juan, Argentina \\ iD https://orcid.org/0000-0001-6410-1159 \\ aperez@unsj.edu.ar
}

DOl: https://doi.org/10.35622/j.rg.2022.01.005

Recibido: 22/11/2021 Aceptado: 16/02/2022 Publicado: 16/02/2022

PALABRAS CLAVE

aprendizaje colaborativo, competencias transversales, entrenamiento intensivo, permanencia, virtualidad.

\section{KEYWORDS}

collaborative learning, transversal skills, intensive training, permanence, virtuality.
RESUMEN. A raíz de la pandemia COVID-19 y la continuidad del cursado en modalidad virtual, surgieron obstáculos como la ausencia de prácticas, la dificultad de integración estudiantil y la deserción. En el marco de un proyecto de jóvenes investigadores sobre estrategias de articulación y permanencia en la Universidad Nacional de San Juan, este artículo presenta una experiencia de entrenamiento intensivo en competencias transversales para estudiantes de las carreras de Bioingeniería e Ingeniería Electrónica. Se expone una revisión bibliográfica de experiencias competitivas de aprendizaje y se describen las opiniones de los 60 participantes. Se analizan aciertos y desaciertos para investigadores que pretendan realizar experiencias similares. Se enfatiza la necesidad de trabajar sobre el manejo de conflictos, la comprensión de consignas y la tolerancia a la frustración, como emergentes de esta propuesta. Las conclusiones reflexionan sobre la posible incidencia que los espacios alternativos de aprendizaje colaborativo pueden tener sobre el aprendizaje y la permanencia.

ABSTRACT. As a result of the COVID-19 pandemic and the continuity of the virtual course, obstacles arose, such as the absence of internships, the difficulty of student integration, and dropouts. Within the framework of a project of young researchers on articulation and permanence strategies at the National University of San Juan, this article presents an intensive training experience in transversal competencies for students of Bioengineering, and Electronic Engineering careers. A bibliographic review of competitive learning experiences is presented, and the opinions of the 60 participants are described. Successes and mistakes are analyzed for researchers who intend to carry out similar

\footnotetext{
${ }^{1}$ Doctora en Educación de la Universidad Católica de Cuyo. Correspondencia: mseminara@unsj.edu.ar
} 
experiences. As emerging from this proposal, the need to work on conflict management, understanding of slogans, and tolerance for frustration is emphasized. The conclusions reflect on the possible impact that alternative collaborative learning spaces can have on learning and permanence.

\section{PALAVRAS-CHAVE}

aprendizagem colaborativa, competências transversais, formação intensiva, permanência, virtualidade.
RESUM0. Em decorrência da pandemia do COVID-19 e da continuidade do curso virtual, surgiram obstáculos como a ausência de estágios, a dificuldade de integração dos alunos e a evasão. No âmbito de um projeto de jovens investigadores sobre estratégias de articulação e permanência da Universidade Nacional de San Juan, este artigo apresenta uma experiência de formação intensiva em competências transversais para alunos das carreiras de Bioengenharia e Engenharia Electrónica. Uma revisão bibliográfica de experiências de aprendizagem competitivas é apresentada e as opiniões dos 60 participantes são descritas. Sucessos e erros são analisados por pesquisadores que pretendem realizar experiências semelhantes. A necessidade de trabalhar na gestão de conflitos, compreensão de slogans e tolerância à frustração, como emergente desta proposta, é enfatizada. As conclusões refletem sobre o possível impacto que espaços alternativos de aprendizagem colaborativa podem ter na aprendizagem e na permanência.

\section{INTRODUCCIÓN}

A partir de la emergencia sanitaria ocasionada por la pandemia COVID-19 y la continuidad del cursado en modalidad virtual, surgieron dificultades entre las que se destacan la ausencia de prácticas, la dificultad de integración entre estudiantes y la deserción. Al respecto, Manzano et al. (2021) destacan que el compromiso estudiantil con las actividades académicas disminuyó notablemente durante el confinamiento. Por otro lado, esta crisis ha enseñado a las sociedades que el trabajo en materia de integración social aún está muy lejos de lo deseable, no sólo se necesitan estrategias para proveer dispositivos electrónicos a colectivos vulnerables sino, mejorar sus posibilidades de integración laboral (García, 2021).

Dichos obstáculos se sumaron al actual requerimiento de incorporar en los planes de estudio las competencias transversales en la formación universitaria. Al respecto de esta última, Cazorla (2017) especifica que, aunque una de las metas comunes de las instituciones de Educación Superior es la de preparar a los alumnos para su futura incorporación al mundo profesional, en ocasiones la conexión universidad-medio laboral falla, pudiendo observarse en los egresados una formación excesivamente teórica y escasa preparación para la investigación y la creatividad, así como para la integración en equipos de trabajo.

Pese a los numerosos trabajos de investigación que se observan en la literatura, se puede apreciar que las reformas integrales y transversales son necesarias para alcanzar el desarrollo de las competencias y el modo de impulsarlas, aún no ha sido alcanzado (OECD, 2017 a.2017 b).

En tal sentido, para el caso de Ingenierías, en el año 2018 se establece la propuesta al CIN de estándares de segunda generación para la acreditación de carreras. Donde el libro rojo especifica que la formación del ingeniero debe poner foco en el estudiante y fortalecer las competencias genéricas y específicas del graduado (CONFEDI, 2018). En esta línea, Mastache y Goggi (2017) exponen que una sólida formación envuelve desempeñarse de manera flexible en situaciones profesionales complejas, desarrollando la capacidad de resolución de problemas, a partir de la articulación entre el pensamiento divergente 0 ingenio y el lógicomatemático. Sostienen que esto sólo es posible a partir de una enseñanza que genere actividades centradas en el aprendizaje, capaces de desarrollar la comprensión y de desplegar procesos que favorezcan la reflexión 
retrospectiva, el conocimiento generador y el aprendizaje a partir de la experiencia. Consideran que el desafío, entonces, es pensar una enseñanza que proponga experiencias formativas, que se constituyan en oportunidades para el desarrollo de sujetos autores de su aprendizaje y de su desarrollo profesional. Para ello, se requieren propiciar y sostener estrategias pedagógico-didácticas tales como de la articulación de contenidos de diferentes asignaturas.

En el marco de un proyecto de jóvenes investigadores sobre estrategias de articulación y permanencia en la Facultad de Ingeniería de la Universidad Nacional de San Juan, el 13, 14 y 15 de mayo de 2021 se llevaron a cabo las primeras competencias de innovación del departamento de electrónica y automática de la Facultad de Ingeniería de la Universidad Nacional de San Juan. Las mismas tuvieron como eje el desarrollo de las competencias transversales y el trabajo colaborativo. Esto podría implicar un beneficio en relación a la permanencia e integración de los estudiantes, especialmente en época de confinamiento.

Según Revelo et al., (2018) el trabajo colaborativo, en un contexto educativo, constituye un modelo de aprendizaje interactivo, que invita a los estudiantes a construir conjugando esfuerzos y talentos. Asimismo, les permitan lograr las metas consensuadas. Más que una técnica, es una filosofía de interacción y una forma de trabajo, que implica una fuerte interdependencia y respeto a las contribuciones individuales de los miembros del grupo, que se caracterizan por tener una heterogeneidad de habilidades.

El presente artículo describe resumidamente el modo en que se diseñó e implementó la competencia. A continuación, se sintetizan algunas de las opiniones de los estudiantes sobre la experiencia, las cuales fueron recabadas en tres períodos: antes, durante y después de la misma. Por último, en base a las dificultades y aciertos detectados, se discute sobre el objetivo principal del trabajo, indagar si estos espacios alternativos de aprendizaje contribuyen efectivamente en el desarrollo de competencias transversales y la integración entre estudiantes en pos de la permanencia.

\section{MÉTODO Y MATERIALES}

El estudio fue exploratorio cualitativo descriptivo. Se consideró exploratorio ya que se pretendió abrir nuevas alternativas ante los obstáculos surgidos a raíz de la pandemia Covid-19, así como ante la necesidad de desarrollar las competencias transversales en la formación de estudiantes universitarios. Por otra parte, puede categorizarse como transversal ya que los datos fueron recabados en un período puntual de tiempo.

Las técnicas utilizadas comprendieron la revisión de la literatura y el análisis de cuestionarios. En relación a la primera, se tomaron 80 artículos de revistas científicas indexadas a partir de términos claves tales como competencias transversales, entrenamiento intensivo, competencias de aprendizaje y trabajo colaborativo, entre otros. De entre estos, se prefirieron 50, aquellos realizados por autores hispano hablantes a fin de poder observar experiencias en contextos similares al propio.

En cuanto a la segunda técnica, el cuestionario, aquí se dividió en 3 secciones a fin de ser aplicado antes, durante y el día después del evento competitivo. Los datos se analizaron de manera cuantitativa porcentual y descriptiva. A partir de dicho análisis, se abre el debate que conduce a reflexionar sobre la posible eficacia de estos espacios para la integración estudiantil, el desarrollo de competencias transversales y la permanencia. 
La muestra estuvo conformada por 60 estudiantes que voluntariamente decidieron participar. Fueron divididos en 12 grupos de 5 integrantes cada uno. El 54,2\% de los participantes pertenecía a la carrera de Bioingeniería y el restante $45,8 \%$ a la carrera de Ingeniería Electrónica. Todos los grupos se constituyeron con alumnos de ambas carreras y al menos un estudiante de primer o segundo año, a fin de propiciar la integración entre estudiantes más allá del objetivo de la resolución del desafío propuesto en la competencia.

\section{RESULTADOS}

\subsection{La revisión bibliográfica y la experiencia del entrenamiento intensivo en competencias transversales en la Universidad Nacional de San Juan}

Según la bibliografía, uno de los retos actuales dentro de la educación superior es la necesidad urgente de cambiar el enfoque de enseñanza para proveer a los estudiantes destrezas adicionales que les permitan analizar y resolver problemas reales. La UNESCO (2016) organiza las competencias transversales en 6 grupos: pensamiento crítico y de innovación, competencias interpersonales, competencias intrapersonales, competencias relativas al ser ciudadanos globales, manejo de la información y otras. En esta línea, la asociación de Colegios Americanos en base a encuestas realizadas en el año 2013 a distintos empleadores, indicó que el 95\% preferían a graduados universitarios capaces de contribuir a innovaciones en su lugar de trabajo y un 93\% estuvo de acuerdo con que más que el título, lo que resulta imprescindible es que el candidato demuestre capacidad de pensamiento crítico, resolución de problemas complejos y comunicación efectiva (Calco \& Veeck, 2015 cit. por Vivanco \& Castillo, 2018).

Según Rodríguez et al., (2017) de acuerdo con el reporte NMC Horizon report 2016: Higher Education Edition, en las próximas décadas se pueden predecir algunos cambios en la educación superior. Dentro de las tendencias a corto plazo se espera que en las instituciones de educación se introduzcan progresivamente los diseños de aprendizaje que mezclan diversos medios y estrategias, así como nuevas formas de medir el aprendizaje.

Es claro entonces que las competencias son tema prioritario en las agendas educativas universitarias y que el desarrollo de aquellas denominadas transversales resulta imprescindible para las carreras de ingeniería. Donde los egresados han manifestado algunas debilidades al momento de su inserción laboral (Sukanto \& Effedi, 2016 cit. por Pizarro, 2021). Para Cedeño et al., (2018) las competencias transversales son aquellas que se articulan en la lógica de la interacción del profesional en su actuación específica y básica. Para su desarrollo, según Tobón (2013 0p. Cit.) debe estimularse la resolución de problemas pertinentes del contexto mediante acciones de direccionamiento, planeación, actuación y comunicación de los productos logrados. En la misma línea, Boix \& Burset (2011) destacan que trabajar por competencias significa que el alumno debe entender el aprendizaje 
como un circuito multidireccional donde tiene que tomar la iniciativa y estimular la capacidad crítica, ética, creativa y sensible en la gestión de su aprendizaje a todos los niveles para favorecer su formación integral. En este caso, el profesor es un guía o despertador de curiosidades que acompaña al alumno en este trayecto.

De acuerdo con Aristulle y Paoloni (2019) para que una persona demuestre competencia en una determinada tarea, función o rol, no sólo necesita dominar una serie de conocimientos conceptuales, procedimentales y actitudinales, además deberá estar motivada para actuar y contar con un contexto favorable para la actuación que se pretende llevar a cabo.

Zabala y Arnau (2008) señalan que no hay una metodología especifica para la enseñanza de las competencias, pero sí existen condiciones para su desarrollo. Entre estas señalan que el aprendizaje debe ser activo y significativo para el estudiante, así como provocar conflicto cognitivo para promover el pensamiento crítico. También consideran que las estrategias tienen que ser graduales en cuanto a la complejidad, de manera que resulten retos asumibles y tienen que permitir la autonomía y la multiplicidad de resultados. Asimismo, debe promoverse el trabajo interdisciplinario. En la misma línea, Leboyer hace ya dos décadas afirmó que "[...] las experiencias obtenidas de la acción, de la asunción de responsabilidad real y del enfrentamiento a problemas concretos, aportan realmente competencias que la mejor enseñanza jamás será capaz de proporcionar” (1997, p. 27 cit. por Martínez \& González, 2019).

Muchos estudios han utilizado como metodologías las Hackathones (Rodríguez et al., 2019; Mejía et al., 2018; Terán et al., 2020, Ramírez \& Duarte, 2020; Ramírez et al., 2020; Vázquez \& López, 2018, Rayna \& Striukova, 2021, La Place et al., 2017, Larrondo et al., 2021, entre otros). Pese a que la palabra ha solido desvirtuarse, un hacker es una persona curiosa y dedicada que intenta resolver un desafío y ponerlo al servicio de los demás, utilizando para ello sus conocimientos técnicos. El término tiene su origen en los laboratorios del Instituto Tecnológico de Massachusetts y, aunque originalmente ligado al manejo de computadoras y seguridad de los sistemas, se ha extendido a diferentes áreas de interés. En la misma línea, los encuentros en formato hackathon o competencia intensiva, trasladan la idea de un encuentro tecnológico exigente e ininterrumpido a cualquier tipo de evento que implica a personas de distintos sectores en la resolución creativa focalizada de un reto mediante una experiencia colaborativa.

Tal como lo afirma Nandi y Mandernach (2016) el desarrollo de los Hackathons provoca que los estudiantes se motiven, pero la pregunta es si efectivamente colaboran en el desarrollo de las competencias. Pese a que no hay afirmaciones concretas sobre esta pregunta, varios investigadores se han encargado de revisar sus elementos esenciales. En tal sentido, Gardó y Riera (2020) revisaron la bibliografía contrastándola con la propia 
experiencia como organizadores del EDhack Raval, el primer hackathon educativo comunitario de Cataluña. Sus resultados apuntaron a la valorización de la libertad del proceso creativo, la generación de redes comunitarias y el aprendizaje social por encima de la creación de soluciones y advirtieron sobre la necesidad de cerciorarse de no caer en la organización de eventos que promocionen el solucionismo ni el simplificar un reto social complejo en una solución creada en pocas horas. Subrayan que no hay evidencias ni reflexión suficiente para concretar el valor diferencial que aportan las competencias a la educación. Lo mismo sostienen Saldarriaga y Guzmán (2018) quienes además destacan similares beneficios. Estas competencias permiten practicar intensivamente el aprendizaje basado en problemas, construyendo una solución siguiendo el modelo Design Thinking, el cual centra su eficacia en entender las necesidades reales de los usuarios (Silver et al., 2016; Vivanco et al., 2018). Es una metodología diseñada en Stanford se popularizó entre empresas y fue tomada por los gobiernos también, para abordar desafíos públicos. El proceso consiste en cinco etapas que son: empatizar, definir, idear, prototipar y testear (Acevedo \& Dassen, 2016).

En tal sentido, desde el Departamento de Electrónica y Automática de la UNSJ, donde se dictan las carreras de Bioingeniería e Ingeniería Electrónica, se diseñó una Competencia de Innovación, con el objetivo de propiciar entrenamiento intensivo en competencias transversales. Basada en el Rally Latinoamericano de Innovación, se desarrolló durante 3 días.

Durante los dos primeros, los estudiantes participaron en 5 talleres formativos, a saber: creatividad, herramientas para ser emprendedor, comunicación efectiva, modelo de negocios y uso colaborativo de la plataforma a través de la cual se desarrolló el evento. El tercer día se llevó a cabo la competencia propiamente dicha. Se partió de la presentación de un desafío real por parte de un organismo estatal, el cual tenía como eje la inclusión de todas las personas en el sistema de transporte público de la provincia. Los alumnos debían resolverlo en equipos integrados por estudiantes de ambas carreras, durante 12 horas continuadas. Implicaba la articulación de conocimientos de diferentes asignaturas para encontrar una solución innovadora, la cual fue evaluada por un jurado interdisciplinario según una rúbrica realizada para este evento y basada en criterios como originalidad, tecnología utilizada, posible impacto social, calidad de la presentación y viabilidad de concreción de la propuesta, entre otros. El premio para los ganadores se vinculó con la posibilidad de incubación de la idea, así como una capacitación para todos los participantes.

La comunidad implicada abarco los 60 estudiantes, un equipo de organización de docentes y miembros de las asociaciones estudiantiles, 12 docentes tutores colaboradores y 7 miembros del jurado, además de 5 disertantes de talleres. El jurado comprendió un equipo interdisciplinario formado por 2 miembros de empresas, 2 docentes 
de las carreras mencionadas, una autoridad perteneciente a la Facultad, un egresado y un miembro de una asociación de discapacidad vinculada con el desafío.

Se llevó a cabo en formato virtual, dada la situación sanitaria, y procuró establecer condiciones para fomentar la integración entre estudiantes de diferentes años y de ambas carreras, considerando que el aislamiento ha obstaculizado la interacción natural. Cabe destacar que la competencia se realizó un día sábado para no superponerse con asignaturas ni con horarios laborales de muchos de los estudiantes.

Como primera experiencia, además, pretende promover el cambio en la cultura institucional hacia la participación de los docentes en un clima de visión compartida sobre las necesidades de estimulación del proceso de implementación de las competencias.

\subsection{Las opiniones surgidas a partir de los cuestionarios diseñados y aplicados para esta experiencia}

\section{a. Respuestas estudiantiles previas a la competencia}

El 83,1\% indicó no haber participado antes en una experiencia de innovación, mientras el 11, 9\% dijo que participó una vez y el restante 5\% más de una vez.

El 71,2\% señaló no tener experiencia en trabajo en equipo, mientras el 28,8\% sí. De ese último porcentaje, las experiencias más importantes referían a la participación en: olimpiadas, agrupaciones estudiantiles, tutorías de pares, trabajo no académico, trabajos prácticos de la carrera que cursaban, trabajos finales de la escuela de nivel medio, ferias de ciencias, competencias de contenidos educativos en internet, deportes, dinámicas grupales, prácticas de laboratorio, ayudantías docentes, pasantías, grupos religiosos y voluntariados, etc. Dentro del porcentaje que señaló contar con experiencia en trabajo en equipo, el 67,8\% indicó no tener experiencia en trabajo remoto y el 32,2\% sí.

En relación a los principales intereses en participar en la competencia, los estudiantes manifestaron: A. Intereses vinculados a las competencias académico-disciplinares: aprender, afianzar conocimientos, participar de los talleres formativos previos, iniciar una destreza nueva, poner a prueba la creatividad y habilidades, obtener conocimiento, aprovechar el aprendizaje empírico, conocer más sobre la carrera, conocer la propia capacidad para aplicar conocimientos, descubrir diferentes maneras de resolver problemáticas. B. Intereses vinculados a las competencias sociales: conocer estudiantes avanzados en la carrera, trabajar interdisciplinariamente, aprender a trabajar en equipo, participar con compañeros de otra carrera y otros años, pasar una linda experiencia en equipo, generar nuevos vínculos, interactuar y expandir la mente, divertirse, generar ideas buenas 
ante problemáticas importantes para la sociedad, incentivar a los ingresantes, conocer gente del sector productivo local, compartir conocimientos. C. Intereses vinculados al futuro profesional: probar actividades relacionadas con el ámbito laboral, adquirir experiencia, capacitarse, probar la capacidad de trabajar bajo presión, introducirse en el desarrollo de proyectos, adquirir nuevas herramientas y experiencias complementarias a la formación dentro de un equipo, probar las habilidades adquiridas para resolver problemas de ingeniería estando cerca de la graduación, adquirir ideas para desarrollarlas a futuro, tener un panorama de las demandas actuales de las empresas D. Intereses extrínsecos: obtener reconocimiento y premio, ganar, competir, que sea un disparador de ideas y un generador de proyectos para presentar en otras competencias.

En relación a cómo habían sido sus procesos para crear algo previamente, los participantes detallaron estrategias tales como: crear una hoja de ruta rápida, indagar si la idea ya fue planteada para mejorarla o cambiar de dirección buscando, generar ideas en equipo y hacer una presentación general, conocer debilidades y fortalezas de los integrantes del equipo, realizar una actividad o juego que permita conocerse, informarse sobre el problema a resolver, generar lluvias de ideas, dividir el proyecto en disciplinas y distribuirlas a cada integrante según fortalezas y experiencias, plantear estructuras básicas y luego ir mejorándolas, desarmar el problema e identificar qué partes pueden resolverse con conocimientos ya adquiridos para saber cuáles se necesitan incorporar, unir soluciones pequeñas para lograr la solución total, empezar desde lo básico hasta lo complejo utilizando softwares y simuladores para montar y probar, analizar todos los aspectos del problema, consultar a expertos que puedan orientar sobre la viabilidad de la idea, seguir un plan de ideación-análisis-factibilidadplanificación-ejecución y evaluación, etc.

\section{b. Respuestas estudiantiles obtenidas durante la competencia}

Se les pidió que nombraran al equipo y comentaran cómo eligieron el nombre para conocer como habían comenzado a interactuar y generaran pertenencia con el mismo. Luego se les solicitó que relataran cómo fue sucediendo el proceso de solución de la idea/creación y las dificultades surgidas.

También se indagó sobre cómo se organizaron para trabajar en grupos y si establecieron roles. El 16,7\% dijo que no, mientras que el resto sí, aunque luego de un debate conjunto inicial. Señalaron que los roles fueron alternados y dinámicos, con actitud de escucha y debate constante. En general, manifestaron que a medida que se requería cerrar una actividad en particular, quien se sintiera cómodo para desarrollarla se ofrecía.

Entre las dificultades que observaron, mencionaron: carecer de conocimientos técnicos suficientes, comprender el problema/desafío planteado, elegir entre las opciones realizables que proyectaban, sintetizar lo realizado en 
3 páginas como lo exigían las condiciones referidas al informe final y dificultades en la realización del video. Ningún grupo señaló obstáculos sobre el trabajo en equipo ni en elaborar una idea realmente innovadora. Ante los conflictos percibidos señalaron haber consultado a los docentes o a un experto, así como a otros grupos, frente a lo cual pudieron obtener una visión más amplia de la problemática y una perspectiva diferente.

En cuanto a los talleres previos, comentaron que fueron de gran utilidad y que, para una próxima oportunidad, sumarían algún taller sobre trabajo en equipo y sobre herramientas audiovisuales. También plantearon la inquietud de poder contar distintas historias sobre el nacimiento de las empresas y la mecánica para lograr esto en Argentina.

En relación a las sugerencias para una próxima experiencia, destacaron que las encuestas de opinión fuesen orales, que la redacción del desafío y de las bases fuese más clara, que los talleres de formación fuesen más extensos, que el cronograma estuviese pautado con mayor anticipación, que hubiese sólo un medio de comunicación para no dispersar la información, que se les preguntara durante la competencia cómo iban trabajando de manera más frecuente y poder trabajar en formato presencial.

\section{c. Respuestas estudiantiles post experiencia}

Se les preguntó cómo calificarían su integración al equipo de trabajo, a lo que todos los grupos comentaron que fue muy buena a pesar de que varios de ellos trabajaron de manera remota y de que muchos no se conocían previamente. Señalaron haber observado gran receptividad a las opiniones de otros compañeros y que los líderes de cada equipo fueron democráticos y rescataron las fortalezas principales de cada uno de los integrantes.

En relación a la propuesta de mencionar el mejor y el peor aspecto o hecho experimentado durante la experiencia, describieron que lo mejor fue crear nuevos vínculos, disfrutar el proceso y la gratificación de entregar el resultado, así como realizar el video. También señalaron el gran compromiso manifiesto por todos los integrantes del equipo. En cuanto a lo que no les gustó, señalaron discusiones surgidas al final de la competencia y falta de compañerismo por parte de algunos grupos al proponer eliminar a los grupos que no habían cumplido de manera exacta con el tiempo y forma de entrega del video e informe. Indicaron que les resultó difícil la redacción del informe y que observaron gran estrés, dificultades de organización y escasez de tiempo.

Por último, se les consultó si volverían a participar. La totalidad de los grupos dijo que sí ya que la experiencia les otorgó apertura en ideas, posibilidad de conocer nuevos compañeros y los motivó a continuar la carrera, así 
como la oportunidad de aprender nuevas herramientas y habilidades, lo cual consideraron esencial en un momento en que la situación sanitaria ha dificultado la interacción. No menos importante, destacaron que había sido una experiencia divertida que además los preparaba para la inserción laboral futura. La dificultad que comentaron es que generalmente la carga de estudio es tan pesada que se dificulta para ellos participar en actividades fuera de lo pautado en las asignaturas.

Sin embargo, luego de conocer la puntuación del jurado y proclamar la resolución de las dos ideas que resultarían ganadoras, se suscitaron conflictos entre los grupos y para con la comisión de organización. Varios participantes manifestaron que se sentían frustrados y no volverían a participar en este tipo de actividades, solicitando explicaciones sobre por qué su idea no resultaba ganadora. En tal sentido puede reconocerse que la idea de un evento competitivo es muy atractiva para algunos participantes, pero para otros puede aparejar estrés y sensaciones negativas. Al respecto, puede acordarse con Sastry (2014) en que las experiencias que fomentan la competencia no necesariamente son eficaces, ya que la innovación requiere reflexión, iteración y colaboración. Por el contrario, una carrera cronometrada en línea recta puede evitar que se asuman riesgos y se intercambie información.

Este último evento de malestar condujo a la necesidad de reflexionar, tal como se propone en el siguiente eje del escrito, si este tipo de experiencias resultan favorables para el aprendizaje y la permanencia, si el conflicto es parte del proceso natural de creación o si debe proponerse otro formato para los espacios alternativos de aprendizaje. También es posible derivar la pregunta sobre qué sucede con la tolerancia a la frustración de los estudiantes que recibe hoy la Universidad.

A futuro se espera realizar una sesión luego de 6 meses culminada la experiencia para conocer qué aprendieron del proyecto, si el proceso de creación fue distinto al que realizaban previo a la competencia, si continúan reuniéndose sólo por el lazo social generado, si no se reúnen más o si incluso se siguen vinculando para construir nuevas ideas juntos, así como qué aprendieron de sí mismos en relación a si descubrieron nuevas habilidades fortalezas, debilidades sobre sí mismos y si pudieron capitalizar eso que descubrieron. En suma, qué impacto tuvo la experiencia a mediano plazo.

\section{DISCUSIONES}

Es claro que estas experiencias colaborativas se basan en una filosofía que no es nueva. La concepción de aprendizaje social y humanista de Rousseau en 1762, el aprendizaje experiencial de Dewey de 1938 con su vertiente de horizontalidad, la desescolarización de Illich de los 70, la pedagogía autónoma de Montessori de 
Ios 60, la perspectiva sociocultural de Vygotski de 1978, la educación maker de Papert de los 90 y muchas otras, son fundamentos de las mismas. El valor de la práctica compartida entre los participantes, entonces, resulta indudable.

Algunos investigadores más recientes afirman que los espacios colaborativos de aprendizaje benefician el rendimiento, otros que colabora en bienestar personal, otros que influye positivamente en el logro de aprendizajes cognitivos, procedimentales y actitudinales, sobre la autonomía y la responsabilidad (Meroño et al., 2021; Palomera et al., 2017; Vargas et al., 2020; González et al., 2013). Otros como Barrett et al., (2013 cit. por Perea et al., 2017) consideran que estos espacios alternativos donde el aprendizaje tiene lugar, ejercen un impacto directo sobre el aprendizaje en sí, de modo que el éxito y progresión de la enseñanza, dependen en gran modo de esos espacios. En añadidura, Brugué et al., (2014 cit por Paonessa, 2019) destacan que la innovación no se trata sólo de incidir en procesos, sino que propician el replanteo de la propia naturaleza de los problemas, modificando la forma de entender, abordar y transformar la realidad.

Por el contrario, Gardó y Riera (2020) señalan que un excesivo énfasis en la eficacia de las soluciones creadas y un enfoque hacia la victoria, dañan el aprendizaje, la colaboración e incluso, el proceso de innovación. Sin embargo, destacan que, si se va más allá de la solución creada, las competencias sirven para visibilizar temas importantes y tejer redes comunitarias a su alrededor. Ayudan a poner en práctica herramientas de colaboración y creación sin miedo al error, implicando a las personas desde la autonomía.

En relación a la experiencia propiciada desde la Universidad Nacional de San juan, cabe destacar que existieron numerosos aspectos positivos tales como los talleres formativos previos, que fueron calificados como enriquecedores por la totalidad de los participantes, e incluso propiciaron la motivación por aprender acerca de nuevas temáticas. Esta motivación se vio reflejada en la sugerencia de los estudiantes acerca de incluir, en una próxima edición, talleres sobre edición de videos y oratoria.

Por otra parte, entre los obstáculos se destaca que faltó una instancia inicial de aclaración detallada del desafío por parte del organismo o empresa que lo sugirió, el cual fue divulgado a modo de consigna escrita. Otra limitación fue que las bases de la competencia contenían algunas sentencias ambiguas que provocaron la necesidad de precisar ciertas reglas y aclaraciones durante la competencia, las cuales podrían haber sido anticipadas a fin de evitar malos entendidos y pérdida de tiempo. Otra restricción fue que, luego de la difusión de los puntajes finales y ganadores, no se ofreció una instancia de diálogo con el jurado o recusación que podría haber resultado enriquecedora. Por último, puede destacarse como limitante el hecho de haber basado 
la evaluación de la experiencia sólo en los cuestionarios y entrevistas mantenidos con los estudiantes, sin haber incluido las opiniones de toda la comunidad docente y empresarial que participó.

Más allá de los aciertos y desaciertos, propios también de una primera edición, el espíritu competitivo de la experiencia condujo a replantear si era beneficiosa para los estudiantes y los objetivos inicialmente propuestos. En este sentido, se acuerda con Moreno (2010) en que suele existir cierta prisa por aplicar una nueva tendencia sin una reflexión conceptual, adoptando esquemas del exterior sin abrir espacios para el análisis y el debate, donde todos los implicados participen y sus voces sean consideradas.

En específico, y tal como se explicitó en un apartado anterior, pese a que se conformó un jurado interdisciplinario y que las creaciones entregadas fueron anónimas para garantizar objetividad en la evaluación, y a pesar de haber creado una rúbrica detallada para la calificación de las propuestas, la proclamación de los ganadores, y "perdedores", modificó por completo el clima colaborativo creado antes y durante la experiencia de aprendizaje. Al respecto, Diaz y Cimavilla (2019) comentan que el debriefing es un proceso de reflexión intencionada que permite construir aprendizajes profundos, pero no es fácil de realizar ya que puede salirse de eje por tensiones como la carga cognitiva, manejo de las emociones, etc. cómo manejar esas situaciones de manera temprana y adecuada, sostienen, es la diferencia entre lograr aprendizajes y generar conflictos.

Por un lado, puede pensarse que el conflicto es parte natural del proceso colaborativo y no tiene por qué ser visto como un defecto o inconveniente de la experiencia. Además, puede considerarse que, si la experiencia pretende el desarrollo de competencias transversales, es necesario estimular la competencia de aprender a convivir con la aceptación del no acuerdo y la habilidad de debatir con otros, tal como lo exponía Gardner enmarcándolas dentro de la inteligencia interpersonal (1994 cit. por García et al., 2016). En tal sentido esta propuesta ha resultado enriquecedora para conocer que es necesario estimular entre los participantes la habilidad de mediación y debate, sin que esto implique una ruptura con otros participantes, grupos o con la experiencia en sí.

Por otra parte, puede pensarse si la dificultad surgió a partir de la técnica utilizada para valorar las producciones de los participantes. En este punto, puede destacarse que, si la evaluación convencional es un problema para buena parte del profesorado universitario, que al día de hoy ni siquiera tenemos resuelto, el desafío se torna mayor cuando lo que se trata de valorar es el aprendizaje por competencias. Más allá de que el debate sobre las técnicas de evaluación adecuadas, lo que debe tomarse como esencia es que hay que transitar el pasaje desde la evaluación del aprendizaje a la evaluación para el aprendizaje y a la evaluación como aprendizaje (Moreno, 2010). En este punto puede tomarse como limitación el no haber indagado si la evaluación del jurado 
resultó formativa para algunos más allá de las emociones surgidas. Además, pueden abrirse interrogantes tales como: ¿resultaría útil añadir la valoración de cada equipo sobre las producciones de los otros? ¿Una valoración cualitativa que igualmente condujera a que algunos ganen y otros pierdan resultaría igualmente conflictiva? ¿Se trata de una falla en la organización o un emergente útil para trabajar sobre la tolerancia a la frustración de los estudiantes, lo cual también repercutiría en la posibilidad de permanencia y aprendizaje en la Universidad?

Respecto a esta dificultad, Gardo y Riera (2020) comentan que es probable que los equipos tengan una tendencia natural a sobrevalorar las creaciones propias, lo cual es comprensible en un evento competitivo emocional. Sugiere equilibrar los momentos de compartir y los de competir. Además, señala acciones que pueden potenciar la colaboración tales como que el premio sea la participación en sí y eliminar elementos que discriminen la valoración de los equipos y sus soluciones (premios, puntuaciones, etc.). Sería interesante ver qué efectos tendría sobre la motivación de los participantes el hecho de prescindir de las estrategias de competición.

Autores centrados en la evolución de los espacios competitivos sociales, como Blikstein y Worsley (2016) 0 Hope at al. (2019) insisten en concebirlas como espacios informales que huyan del intelectualismo. Donde el objetivo es aprender desde la creación y la colaboración. Además, es posible acreditar que el logro de la experiencia no se limita guarda a las producciones allí culminadas, sino a la motivación y debate que se impulsa a partir de allí.

Lo cierto es que el trabajo recién comienza. Varios estudios observan a los espacios de innovación como parte fundamental de la redefinición de la academia a futuro (González et al., 2014; Pascale \& Resina, 2020). En esta línea, consideran que inciden en el intento por que las instituciones sean orgánicas, es decir, que vayan adaptándose al medio. Al respecto Pascale y Resina (2020) subrayan que, como las instituciones suelen ser reticentes a los cambios, este movimiento implica un esfuerzo gradual y constante.

Lo descripto en este artículo sólo rescata el debate y elementos esenciales para quienes deseen continuar la investigación y realizar experiencias similares. Sin embargo, todavía no se puede evaluar su logro ya que a diferencia del desempeño académico que puede ser medido en un momento puntual, tal como señalan Molleda et al (2011) las competencias representan una combinación dinámica de conocimiento, comprensión y habilidades. Éstas se consiguen a lo largo de varios cursos y se adquieren en diferentes niveles. Dicho de otro modo, el que se diga que las competencias se pueden aprender no quiere decir que se logren aprendiendo algo específicamente ni como consecuencia de tener una experiencia delimitada Moreno (2010). 
Respecto a la medición del impacto del aprendizaje sobre la permanencia, la investigación es aún escasa y aún más complicada, por la complejidad inherente al fenómeno de la deserción. En tal sentido, Aparicio ha trabajado tanto el vínculo de la formación con el mundo del trabajo, así como la deserción en su máxima profundidad, en estudios que por su calidad a nivel internacional merecen ser consultados (2005, 2011 a, 2011 b, 2015, 2018).

Queda también por definir cómo dar continuidad a las ideas elaboradas e indagar si esta experiencia produce que las ideas allí gestadas se sigan construyendo, con lo cual podríamos a futuro decir que es una buena experiencia.

\section{CONCLUSIONES}

Este artículo presentó una experiencia de entrenamiento intensivo en competencias transversales desarrollada en la Universidad Nacional de San Juan para las carreras de Ingeniería Electrónica y Bioingeniería. Como estrategia propiciada a raíz de la pandemia COVID-19 y la continuidad del cursado en modalidad virtual, la cual aparejó obstáculos como la ausencia de prácticas, la dificultad de integración estudiantil y la deserción.

En primer lugar, se presentó una revisión bibliográfica acerca de las competencias o hackáthones que estimulan el aprendizaje colaborativo. Subrayando las posiciones de autores que afirman sus ventajas y posibles desaciertos.

A continuación, se describieron las opiniones de los 60 participantes, analizando aciertos y obstáculos para investigadores que pretendieran realizar experiencias similares, y se enfatizó en la necesidad de trabajar sobre el manejo de conflictos, la comprensión de consignas y la tolerancia a la frustración, como emergentes de esta propuesta. Asimismo, los obstáculos permitieron abrir nuevos interrogantes que esperan formar parte de próximas investigaciones.

A partir de dicha exposición y en líneas generales, puede apreciarse que la experiencia fue positiva. Ésta permitió la interacción entre estudiantes y para con los docentes, así como la aplicación práctica de contenidos dictados en diversas asignaturas. También posibilitó la emergencia de conflictos que permitieron observar necesidades latentes en las carreras, tales como la dificultad para la comprensión de consignas y la importancia de la interacción entre docentes. En añadidura, el diseño y organización de la misma ha contribuido en la integración entre docentes y alumnos, así como en la estimulación del vínculo necesario entre la Universidad y el ámbito laboral, especialmente considerando esta etapa de virtualidad en la cual los vínculos se han visto modificados y, en algunos casos, se han dificultado por la ausencia de presencialidad. Sin embargo, aún no se han 
dimensionado sus efectos sobre el aprendizaje de las competencias transversales y la permanencia a largo plazo.

Asimismo, puede considerarse un punto de partida útil e inspirador para un mayor análisis en próximas experiencias alternativas de aprendizaje, pudiendo aportar aspectos esenciales no solo para las competencias transversales sino para cualquier aprendizaje que se decida potenciar.

Por último, el debate entre lo acontecido en la competencia producida, lo percibido por los estudiantes y lo revisado en la literatura, permitió extraer los puntos positivos y limitaciones de los espacios alternativos de aprendizaje con características tanto de competición como de colaboración, y rescatar el valor del conflicto dentro de los procesos de innovación en equipos.

En suma, el debate debe continuar, vinculado a la necesidad de reconocer que, aunque en la Universidad efectivamente se están realizando innovaciones, el problema en el fondo es que en general estas acciones no alcanzan a realizarse extendidas en el tiempo y con la suficiente reflexión posterior como para extraer conclusiones sólidas sobre su posible impacto.

\section{Conflicto de intereses / Competing interests:}

Los autores declaran que no incurre en conflictos de intereses.

\section{Rol de los autores / Authors Roles:}

María Seminara: conceptualización, curación de datos, análisis formal, adquisición de fondos, investigación, metodología, administración del proyecto, recursos, software, supervisión, validación, visualización, escritura preparación del borrador original, escritura - revisar \& amp; edición.

Arnoldo Fernández: conceptualización, análisis formal, investigación, metodología, administración del proyecto, recursos, software, supervisión, validación, visualización, escritura - preparación del borrador original, escritura revisar \& amp; edición.

Arnoldo Fernández: conceptualización, análisis formal, investigación, metodología, administración del proyecto, recursos, software, supervisión, validación, visualización, escritura - preparación del borrador original, escritura revisar \& amp; edición.

\section{Fuentes de financiamiento / Funding:}

Los autores declaran que no recibió un fondo específico para esta investigación.

\section{Aspectos éticos / legales; Ethics / legals:}

Los autores declaran no haber incurrido en aspectos antiéticos, ni haber omitido aspectos legales en la realización de la investigación. 


\section{REFERENCIAS}

Acevedo, S. \& Dassen, N. (2016). Innovando para una mejor gestión La contribución de los laboratorios de $\begin{array}{lllll}\text { innovación } & \text { (Nóblica } & \text { técnica } & \text { del } & \text { BID; }\end{array}$ https://publications.iadb.org/publications/spanish/document/Innovando-para-una-mejorgesti\%C3\%B3n-La-contribuci\%C3\%B3n-de-los-laboratorios-de-innovaci\%C3\%B3np\%C3\%BAblica.pdf

Aparicio (2015). Social Competences and Organisational Devices in their Relationship with of University Studens' Retention. In Carmo, M. (2015) (Ed.). Education Applications \& Developments 13. Portugal: InScience Press. Vol. II, Chapter 17, 185-195.

Aparicio, M. (2011a). Table ronde 6. Las competencias sociales ¿las grandes ausentes en la formación secundaria y universitaria? Congreso Internacional de la Red Iberoamericana de Investigación sobre la Calidad de la Educación Superior (RIAICES), Portugal. Du 24 au 26 février.

Aparicio, M. (2005). Les facteurs psychosociaux en relation avec la réussite universitaire et professionnelle. 2e thèse de doctorat. Paris: Université René Descartes, Sorbonne

Aparicio, M. (2011b). Table ronde 5. Factores psicosociales y "competencias sociales" vinculados a logro académico como parámetros de la Calidad de la Universidad. Un estudio en Argentina. Congreso Internacional de la Red Iberoamericana de Investigación sobre la Calidad de la Educación Superior (RIAICES), Portugal. Du 24 au 26 février

Aparicio, M. (2018). Resiliency and Cooperation or Regarding Social and Collective Competencies for University Achievement. An Analysis from a Systemic Perspective European Journal of Social Science Education and Research, 5(3), 123-135.

Aristulle, P. \& Paoloni, V. (2019). Habilidades socioemocionales en las comunidades educativas: aportes para la formación integral de los y las docentes. Revista Educación, 43(2), 1-26. D0l: https://doi.org/10.15517/revedu.v43i2.28643.

González, E., Martínez y Del Rio (2014). El Laboratorio de Innovación en Humanidades Digitales y la redefinición del perfil del humanista y la academia en el siglo XXI. I Jornadas Nacionales de Humanidades Digitales. Asociación Argentina de Humanidades Digitales, Buenos Aires.

Blikstein, P. \& Worsley, M. (2016) Children Are Not Hackers: Building a Culture of Powerful Ideas, Deep Learning, and Equity in the Maker Movement. Makeology (1st ed., Vol. 1). New York: Routledge. 
Boix, R. \& Burset, S. (2011). "Hacia un nuevo paradigma en la enseñanza superior", en Josep Alsina (coord.), Evaluación por competencias en la universidad: las competencias transversales, Barcelona, Octaedro, 12-17.

Cazorla, A. (2017). Hacia Una Universidad De Investigación Desde Una Profesional. En Cazorla, R. (2017) La universidad: motor de transformación de la sociedad. Disponible en: https://digital.cic.gba.gob.ar/bitstream/handle/11746/7058/Carzola\%20De\%20Giusti\%20La\%20universi dad\%20motor\%20de\%20transformaci\%C3\%B3n\%20de\%20la\%20sociedad\%20PDFA.pdf?sequence=1

Cedeño, G., Sánchez, F. \& Alonso, L. (2018). Formación de la competencia de emprendimiento en estudiantes de economía. Didascalia, IX (4), 69-84.

CONFEDI (2018). Libro Rojo: estándares de segunda generación. Disponible en: https://www.ing.unlp.edu.ar/sitio/institucional/difusion/archivos/LIBRO_ROJO_DE_CONFEDI_estandare S_de_segunda_generacion.pdf

Díaz, D. \& Cimadevilla, B. (2019). Educación basada en simulación: debriefing, sus fundamentos, bondades y dificultades. Revista Latinoamericana de Simulación clínica, 1(2): 95-103.

García, F., Ramírez, R., González, A. \& Pisté, S. (2016). ¿Las inteligencias multiples en la educación superior y la inteligencia de una persona se debe medir por la capacidad lógico matemática y lingüística? CULCyT, 13 (59), 325-333.

García, A. (2021). Building resilient schools: Flexischooling, integration and COVID-19. Revista Española De Educación Comparada, (38), 211-227. https://doi.org/10.5944/reec.38.2021.28840

Gardó, H. \& Riera, J. (2020). Descubriendo las hackatones educativas comunitarias: un estudio exploratorio. Aloma, 38 (1), 51-62.

González, C., P. Martín, M.Á. Carbonero, F. Lara (2013). Evaluación por competencias de los estudiantes de Enfermería a través de Aprendizaje Basado en Problemas, Revista Enfermería Universitaria, 10(4), 120124. DOI: $10.1016 / S 1665-7063(13) 72640-X$

Hope, A., D’ignazio, C., Hoy, J., Michelson, R., Roberts, J., Krontiris, K., \& Zuckerman, E. (2019). Hackathons as Participatory Design: Iterating Feminist Utopias. doi: 10.1145/3290605.3300291

La Place, C., Jordan, S., Lande, M. \& Weiner, S. (2017). Los estudiantes de ingeniería aprenden rápidamente en los eventos de hackaton. Conferencia y exposición annual de la 1ra ASEE, actas de la Conferencia, 2017-Junio. 
Larrondo, A.; Marauri, I.; Meso, K.; Pérez, J.A.; Peña, S. (2021). University Teaching Experiences with Sustainable Development Goals (SDG): Promoting Transversal Competencies in Online Journalism. Preprints 2021, 2021030726 (doi: 10.20944/preprints202103.0726.v1).

León Del Barco, B., Mendo, S., Felipe, E., Polo, M. \& Fajardo, F. (2017). Potencia de equipo y aprendizaje cooperativo en el ámbito Universitario, Revista de Psicodidáctica, 22(1), 9-15. DOI: 10.1016/S11361034(17)30038-2

Manzano, D., Valer, A., \& Hortigüela, D. (2021). Sistema Educativo y actuación ante la pandemia de la COVID19: opinión y perspectivas de mejora según los docentes. Revista Española De Educación Comparada, (38), 112-128. https://doi.org/10.5944/reec.38.2021.28771

Martinez, P. \& Gonzáles, N. (2019). El dominio de competencias transversales en Educación Superior en diferentes contextos formativos. Educ. Pesqui, 45. Disponible en: https://doi.org/10.1590/S16784634201945188436

Mastache, A. \& Goggi, N. (2017). El Comité de Mejora: una experiencia colaborativa en Ingeniería. Revista Argentina de Educación Superior, 14, 165- 177.

Mejía, S. P., Hincapié, J. M. M., \& Giraldo, J. A. T. (2018). Un Modelo Sistémico de Innovación para Universidades Regionales: Revisión de Literatura. Opcion, 34(Special Issue 18), 210-238.

Meroño, L., Calderón, A. \& Arias, J. (2021). Pedagogía digital y aprendizaje cooperativo: efecto sobre los conocimientos tecnológicos y pedagógicos del contenido y el rendimiento académico en formación inicial docente, Revista de Psicodidáctica, 26 (1), 53-61.

Molleda, C., Manrique, E., Montoro, T., Sadornil, E., Vivar, A., Pérez, S., Fernández, C., Jiménez, B., Méndez, A. \& Torres, R. (2011). La adquisición de competencias transversales en la universidad. IX Jornades de xarxes d'investigació en docència universitària: disseny de bones pràctiques docents en el context actual / coord. por María Teresa Tortosa Ybáñez, José Daniel Álvarez Teruel, Neus Pellín Buades, 2011, ISBN 978-84-694-9813-2, pág. 527

Moreno, T. (2010). El currículo por competencias en la universidad: más ruido que nueces. Revista de la educación superior, 39 (154).

Nandi, A. \& Mandernach, M. (2016). Hackathons como plataforma de aprendizaje informal. SIGCSE '16: Actas del $47^{\circ}$ Simposio Técnico de ACM sobre Educación en Ciencias de la Computación, 346-351 https://doi.org/10.1145/2839509.2844590 
OECD (2017 b). Collaborative schools, collaborative students, in PISA 2015 Results (Volume V): Collaborative Problem Solving, OECD Publishing, Paris, http://dx.doi.org/10.1787/9789264285521-12-en

OECD (2017a). Collaborative problem solving, PISA in Focus, No. 78, OECD Publishing, Paris, http://dx.doi.org/10.1787/cdae6d2e-en [61]

Palomera, R., Briones, E., Gomez, A. \& Vera, J. (2017). Cubriendo el vacío: mejorando las competencias sociales y emocionales del profesorado en formación, Revista de Psicodidáctica, 22(2), 142-149. https://doi.org/10.1016/j.psicoe.2017.05.005

Paonessa, L. (2019). Diseñando la colaboración: el caso del Hackaton del Gobierno de la. Ciudad Autónoma de Buenos Aires (2012 a 2015). Tesis de maestría. Universidad de san Andrés, buenos aires.

Pascale, P. \& Resina, J. (2020). Prototipando las instituciones del futuro: el caso de los laboratorios de innovación ciudadana (Labic). Iberoamerican Journal of Development Studies 9(1):6-27. DOI: 10.26754/ojs_ried/ijds.437

Perea, E., Berlanga, V. Y Ruiz, C. (2017). Actividades emprendedoras y espacios alternativos. Desarrollo de competencias durante un hackathon day. EA, Escuela abierta, 20, 47-61. doi:10.29257/EA20.2017.05

Pogacar, K. \& Zizek, A. (2016). Urban Hackathon- Enfoque alternativo basado en información y participativo para el desarrollo urbano. En Ingenieria de procedimiento (vol. 161, 1971-1976). Elsevier

Ramírez, C., Villegas, Y., Vallejos, L., Silva, S. Y Amaral, M. (2020). Modelo de Integración de Grandes Empresas y Startups Mediante Corporate Venture. Revista de investigación de estudios futuros: Tendencias y estrategias, 12 (2), 335-354. doi: https://doi.org/10.24023/FutureJournal/2175-5825/2020.v12i2.448

Rayna, T. \& Striukova, L. (2021) El fomento de habilidades para el 21 st siglo: El papel de los laboratorios y makerspaces Fab. Pronóstico tecnológico y cambio social, 164, marzo de 2021, 120391https://doi.org/10.1016/j.techfore.2020.120391

Revelo, 0.; Collazos, C. A. \& Jiménez, J.A. (2018). El trabajo colaborativo como estrategia didáctica para la enseñanza/aprendizaje de la programación: una revisión sistemática de literatura. TecnoLógicas, 21(41), $115-134$.

Rodríguez, G., Álvarez, M. \& Barraza, R. (2019). Hackathon: experiencia educativa con tecnologías aplicadas al desarrollo de Apps y videojuegos para la solución de problemas medioambientales y de autoempleo. En Tecnologias emergentes y realidad virtual: experiencias lúdicas e inmersivas (pp. 11-39): Egregius. 
Rodríguez, W. R., Denegr, J., Alcocer, M. (2017). Innovación pedagógica: Una oportunidad para la comunidad universitaria en donde todos ganan. Mesa de Reflexión en Innovación Pedagógica y Didáctica. Reflexiones Pedagógicas URosario, 11.

Saldarriaga Salazar, M. E. \& Guzmán González, M. F. (2018). Enseñanza del emprendimiento en la educación superior: ¿Metodología 0 modelo? Revista EAN, 85, 125-142. DOl: https://doi.org/10.21158/01208160.n85.2018.2054

Sastry, A. Y Penn, K. (2014). Fail Better: Design Smart Mistakes and Succeed Sooner. Brighton: Harvard Business Review Press.

Silver, J. K., Binder, D. S., Zubcevik, N. \& Zafonte, R. D. (2016). Healthcare Hackathons Provide Educational and Innovation Opportunities: A Case Study and Best Practice Recommendations. Journal of Medical Systems, 40(7), 1-7. Recuperado de http://doi.org/10.1007/s10916-016-0532-3

Simeone, L., Morelli, N., De Götzen, A. () Chapter 20 - Potential and shortcomings of two design-based strategies for the engagement of city stakeholders with open data

Terán, D. A., Ochoa, A. G., \& Torres, Y. A. (2020). Aprender Enseñando Desde Y Hacia Los Estudiantes. Encuentro Internacional De Educación En Ingeniería. Recuperado a partir de https://acofipapers.org/index.php/eiei/article/view/718

UNESCO (2016). School and teaching practices for twenty-first century challenges: lessons from the Asia-Pacific region, regional synthesis report; 2014 regional study on transversal competencies in education policies. ISBN 978-92-9223-540-6. Available online: https://unesdoc.unesco.org/ark:/48223/pf0000244022

Vargas, K., Yana, M., Perez, K., Chura, W., \& Alanoca, R. (2020). Aprendizaje colaborativo: una estrategia que humaniza la educación. Revista Innova Educación, 2(2), 363-379. https://doi.org/10.35622/j.rie.2020.02.009

Vázquez, J. \& López-García, X. (2018). Actors involved in the consolidation of the interactive documentary. Hipertext.net, [online], 17, 32-42, https://doi.org/10.31009/hipertext.net.2018.i17.04 [View: 18-06-2021].

Vivanco, 0.; Castillo Malla, D. \& Jiménez-Gaona, Y. (2018). Hackathon multidisciplinario: fortalecimiento del aprendizaje basado en proyectos Multidisciplinary. Revista electrónica calidad en la educación superior. Universidad Técnica Particular de Loja, 9 (1).

Zabala, A. \& Arnau, L. (2008). 11 ideas clave: cómo aprender y enseñar competencias. Barcelona: Graó. 